\title{
Empoderamiento de la mujer y habilidades gerenciales en el logro de la responsabilidad social empresarial de las empresas de artesanías de la región Puno - Perú
}

\section{Empowerment of women and managerial skills in achieving corporate social responsibility in handicraft companies from the Puno region - Peru}

Zoraima Julieta Laura-Castillo

Universidad Nacional del Altiplano (UNAP), Facultad de Ciencias Contables

y Administrativas, Escuela Profesional de Administración, Puno, Perú

zilaura@unap.edu.pe

orcid.org/0000-0001-7460-6740

\section{RESUMEN:}

El objetivo de la investigación es determinar la incidencia del empoderamiento de la mujer y sus habilidades gerenciales en la responsabilidad social empresarial de las empresas de artesanías de la región Puno, periodo 2019. El estudio es cuantitativo; con alcance descriptivo, correlacional-explicativo y diseño no experimental-transeccional. La muestra se conformó por 160 mujeres, se aplicó una encuesta procesada en el programa SPSS 22.0 y hoja electrónica Excel. Los resultados muestran que el nivel de empoderamiento de la mujer que pertenece a las empresas de artesanías de la región Puno obtuvo una puntuación de 106.11. Las habilidades conceptuales, grupales, personales y técnicas son las que predominan en las mujeres dentro de las habilidades gerenciales y el impulso al cumplimento de indicadores de responsabilidad social en las empresas de artesanía de la Región Puno se ha dado en dimensiones como comunidad, consumidores-clientes y medio ambiente. Se concluye que con un coeficiente de determinación obtenido por el modelo que es $\mathrm{R} 2=0.383$, significa que el 38,3 $\%$ de la variabilidad de la responsabilidad social empresarial de las empresas, queda explicada por el empoderamiento de la mujer y sus habilidades gerenciales.

\begin{abstract}
:
The objective of the research is to determine the incidence of the empowerment of women and their managerial skills in the corporate social responsibility of handicraft companies in the Puno region, period 2019. The study is quantitative; with descriptive, correlational-explanatory scope and nonexperimental-transectional design. The sample was made up of 160 women, a survey processed in the SPSS 22.0 program and an Excel electronic sheet were applied. The results show that the level of empowerment of women belonging to handicraft companies in the Puno region obtained a score of 106.11. Conceptual, group, personal and technical skills are those that predominate in women within managerial skills and the promotion of compliance with indicators of social responsibility in handicraft companies in the Puno Region has been given in dimensions such as community, consumers- customers and environment. It is concluded that with a determination coefficient obtained by the model that is $\mathrm{R} 2=0.383$, it means that $38.3 \%$ of the variability of corporate social responsibility of companies is explained by the empowerment of women and their managerial skills.
\end{abstract}

PALABRAS CLAVE:

EMPODERAMIENTO,

EMPODERAMIENTO DE

LA MUJER, HABILIDADES

GERENCIALES,

RESPONSABILIDAD SOCIAL

EMPRESARIAL Y ARTESANÍA.
KEY WORDS:

EMPOWERMENT,

EMPOWERMENT OF

WOMEN, MANAGEMENT

SKILLS, CORPORATE

SOCIAL RESPONSIBILITY

AND CRAFTSMANSHIP. 


\section{RÉSUMÉ}

L'objectif de la recherche est de déterminer l'incidence de l'autonomisation des femmes et de leurs compétences en gestion sur la responsabilité sociale des entreprises artisanales de la région Puno, période 2019. L'étude est quantitative; avec portée descriptive, corrélation-explicative et conception non expérimentale-transactionnelle. L'échantillon a été constitué par 160 femmes, une enquête traitée dans le programme SPSS 22.0 et feuille électronique Excel. Les résultats montrent que le niveau d'autonomisation des femmes appartenant aux entreprises artisanales de la région de Puno a atteint 106,11. Les compétences conceptuelles, de groupes, personnels et techniques sont celles qui prédominent chez les femmes dans les compétences managériales et la promotion du respect des indicateurs de responsabilité sociale dans les entreprises artisanales de la Région Puno a été donnée en tant que communauté, consommateurs-clients et environnement. Il est conclu qu'avec un coefficient de détermination obtenu par le modèle qui est $\mathrm{R} 2=0.383$, cela signifie que $38,3 \%$ de la variabilité de la responsabilité sociale des entreprises s'explique par l'autonomisation des femmes et leurs compétences en matière de gestion.
RESUMO:

O objetivo da investigação é determinar a incidência do empoderamento das mulheres e de suas competências gerenciais na responsabilidade social empresarial de empresas de artesanato na região de Puno, período 2019. O estudo é quantitativo; com alcance descritivo, correlacionalexplicativo e desenho não-experimental-transversal. A amostra foi composta por 160 mulheres, aplicou-se uma enquete processada no programa SPSS 22.0 e aplicação de folha eletrônica Excel. Os resultados mostram que o nível de empoderamento das mulheres pertencentes às empresas de artesanato da região de Puno obteve uma pontuação de 106,11. As competências conceptuais, grupais, pessoais e técnicas são as que predominam nas mulheres dentro das competências empresariais e o impulso ao cumprimento dos indicadores de responsabilidade social nas empresas de artesanato da Região de Puno tem se dado em dimensões como comunidade, consumidores-clientes e meio ambiente. Conclui-se que com um coeficiente de determinação obtido pelo modelo que é $\mathrm{R} 2=0,383$, significa que $38,3 \%$ da variabilidade da responsabilidade social empresarial das empresas é explicada pelo empoderamento das mulheres e suas competências gerenciais.

\section{MOTS-CLÉS: \\ AUTONOMISATION DE LA \\ FEMME, COMPÉTENCES \\ MANAGÉRIALES, \\ RESPONSABILITÉ SOCIALE \\ DES ENTREPRISES, \\ ARTISANAT.}

PALAVRAS-CHAVE:

EMPODERAMENTO,

EMPODERAMENTO DA

MULHER, HABILIDADES

GERENCIAIS,

RESPONSABILIDADE SOCIAL

EMPRESARIAL E ARTESANATO. 


\section{INTRODUCCIÓN}

El 25 de septiembre de 2015, los líderes mundiales adoptaron un conjunto de objetivos globales para erradicar la pobreza, proteger el planeta y asegurar la prosperidad para todos como parte de una nueva agenda de desarrollo sostenible. Gamboa-Bernal (2015) y la Organización Internacional del Trabajo (2017) resaltan que dentro de los 17 objetivos esta la igualdad de género, no solo como un derecho humano fundamental, sino la base necesaria para conseguir un mundo pacífico, próspero y sostenible. En ese contexto Caicedo-Muñoz y Solarte-Pazos (2015) refiere al empoderamiento de la mujer, como un proceso interdependiente, que emerge como producto de la acción de ellas sobre los problemas sociales y públicos de su comunidad. Complementa Vargas-Winstanley, Ruiz-Bravo y Clausen-Lizárraga (2018), el empoderamiento femenino se define como la capacidad de tomar decisiones y poder ejecutarlas, HoinleRuthfuss y Gotto (2013) teniendo empleo, autonomía económica y de decisión frente al esposo, sobre sí mismas, su cuerpo y su familia; tener una autoestima alta y tomar la iniciativa en su comunidad y en su familia. Hernandez-Sanchez y Garcia (2008), propone siete dimensiones del empoderamiento, i) participación, ii) temeridad, iii) relaciones externas o sociales, iv) independencia, v) igualdad, vi) la satisfacción social e vii) integridad-seguridad. La CEPAL (2017) y el BID (2015) afirma que las mujeres emprendedoras tienen el potencial de promover el crecimiento económico a través de los nuevos empleos e ingresos que generan con sus micro, pequeños y medianos emprendimientos.

Empero, Gonzáles y Ley (2019) afirma que dirigir un negocio o empresa, no se trata solo de hacer bien las cosas, sino además hacer lo correcto y con calidad, Moreno-Baquedano y Wong (2018) esto significa que no solo debe haber buenos gerentes, también líderes competentes y aptos en cualquier ámbito laboral. Ascón-Villa (2018) plantea que una persona puede llegar a ser un gerente eficaz, realmente justo y organizado, pero sin la habilidad para ser un líder y motivador de sus empleados, otras personas pueden ser líderes altamente eficaces y con grandes habilidades para generar entusiasmo, pero no tiene la suficiente habilidad técnica. Entonces Leiva y Espejel (2017) refiere que las habilidades gerenciales no solo se limitan a un campo cognitivo, más bien son integrales, porque también requieren pasión y constancia.

Apuntando a la integralidad Garcia-Payares, Boom-Carcamo y Molina (2017) propone una clasificación donde considera que las habilidades conceptuales, Aparicio-Valdez y Medina (2015) a las habilidades grupales. NaranjoArango (2015) a las habilidades personales y Garcia-Payares, Boom-Carcamo y Molina (2017) asi como, Gonzáles y Ley (2019) agregan a las habilidades técnicas. Garcia-Solarte, Garcia-Perez de Lema y Madrid (2018) complementan, que estas habilidades, las mujeres poseen en forma natural, pues se encuentra científicamente probado que el hemisferio derecho (emociones) y el hemisferio izquierdo (conocimientos) del cerebro de una mujer se encuentran plenamente conectados, factor a favor, pues esta conexión le permite ser más eficiente a la hora del manejo de recursos y formulación de estrategias.

Hay que destacar que las estrategias hoy en día se traducen en beneficios para las empresas y la responsabilidad social, Herrera-Madueño, Larrán-Jorge y Martinez (2013) es "una expresión que ha entrado con fuerza en los últimos años en la agenda económica y empresarial y, posteriormente, también en la agenda política y social” para favorecer a todas las partes interesadas y no solo a los accionistas. Rojas-Terrazas, Niño-Ramos y Martinez (2017) señalan que el desarrollo de la responsabilidad social empresarial como estrategia, ha permitido hoy en día a la empresa ser más competitiva logrando y demostrando entre otras crear confianza para los consumidores, dado que sus prácticas permiten satisfacer sus necesidades y van dirigidas al bien común. Instituto Ethos (2013), Franco (2006) y IARSE (2008) dimensionan a la responsabilidad social empresarial en: valores y transparencia, público interno, medio ambiente, proveedores, clientes, comunidad y gobierno y sociedad.

En el Perú, en especial en la Región Puno existe una cultura tradicional en artesanía, reflejada en hermosas manifestaciones que se muestran a través de los productos pertenecientes a diversas líneas. Rios (2019) las prendas poseen, inevitablemente, una carga de identidad referida al grupo social a partir del empleo de determinadas técnicas, diseños y colores. Burns (2015) la carga afectiva está relacionada más bien con el recuerdo del artífice, como obsequio o herencia. Forstner (2013) por lo general, es la abuela o la madre tejedoras quienes regalan el arte y secretos del tejido a las nietas e hijas predilectas, la Dirección General de Artesanía del Ministerio de Comercio Exterior y Turismo afirma que, como actividad productiva, posee características definidas, desarrollándose en forma espontánea y empírica y además resalta que las empresas dedicadas al rubro proporcionan un sustento económico a un gran número de personas y hace una enorme contribución a las exportaciones y los ingresos de divisas del Perú. 
En este orden de ideas, la investigación tiene como objetivos específicos: i) determinar el nivel de empoderamiento de la mujer en las empresas de artesanías de la Región Puno, ii) identificar las habilidades gerenciales de la mujer que predominan en las empresas de artesanías de la Región Puno, iii) analizar el cumplimiento de los indicadores de responsabilidad social empresarial en las empresas de artesanías de la Región Puno. Los mismos que han permitido lograr el objetivo general: determinar la incidencia del empoderamiento de la mujer y sus habilidades gerenciales en la responsabilidad social empresarial de las empresas de artesanías de la región Puno.

\section{MÉTODOS}

Ambiente o Lugar de estudio. La investigación se ubica en el departamento de Puno, ubicado al extremo sur este del Perú, políticamente dividido en trece provincias, cuya distribución geográfica permite establecer la zona sur y zona norte. El periodo de estudio comprende los meses de abril y diciembre del año 2019.

Descripción de métodos. La investigación tiene un enfoque cuantitativo; de alcance descriptivo, correlacionalexplicativo y diseño de investigación no experimental. En cuanto a la selección de instrumentos para la variable empoderamiento de la mujer se ha utilizado el instrumento validado por Hernandez-Sanchez y Garcia (2008) que consta de 34 ítems medidos en una escala de Likert donde $1=$ totalmente en desacuerdo; $2=$ desacuerdo; $3=$ ni en acuerdo ni en desacuerdo; $4=$ acuerdo y $5=$ totalmente de acuerdo. Para la variable habilidades gerenciales se ha utilizado un instrumento que consta de 29 ítems medidos en una escala de Likert donde $1=$ nunca; $2=$ casi nunca; $3=$ a veces; $4=$ casi siempre y $5=$ siempre; y finalmente para la variable responsabilidad social empresarial se ha utilizado un instrumento validado por el Instituto Argentino de Responsabilidad Social Empresarial, que consta de 37 ítems, medidos en una escala de Likert donde $1=$ nunca; $2=$ casi nunca; $3=$ a veces; $4=$ casi siempre y $5=$ siempre. Lo anterior aplicado a una muestra probabilística estratificada que consta de 160 mujeres que pertenecen a las empresas de artesanías de la región Puno, distribuidas en las provincias de Puno, Chucuito, Lampa y Melgar (zona sur y norte).

Prueba estadística, para el procesamiento de datos se utilizó el programa SPSS y la hoja de cálculo Microsoft Excel, en las que se establecieron las medidas de tendencia central y las correlaciones respectivas para la regresión lineal múltiple para el cumplimiento de los objetivos.

\section{RESULTADOS}

\section{Nivel de empoderamiento de la mujer de las empresas de artesanías de la Región Puno}

Para el logro de este objetivo se ha tomado como referencia la tabla 1, del instrumento utilizado, la misma que categoriza el nivel de empoderamiento según el puntaje obtenido en un rango menor de 89, de 90 a 104 y mayor de 105. La suma de las dimensiones, participación (27.51), temeridad (13.16), influencias externas (16.70), independencia (10.88), igualdad (11.75), satisfacción social (13.93), seguridad (14.00), ha dado como resultado final una puntuación de 106.11 para la mujer de las empresas de artesanía de la región Puno, resultado que permite inferir que el nivel de empoderamiento de éstas es alto (tabla 2) (figura 1).

\section{Tabla 1. Empoderamiento según el puntaje obtenido}

\begin{tabular}{|c|c|}
\hline Rango & Categoría \\
\hline Mayor de 105 & Empoderamiento alto \\
\hline De 90 a 104 & Empoderamiento medio \\
\hline Menor de 89 & Empoderamiento bajo \\
\hline
\end{tabular}

Fuente: (Hernandez Sanchez y Garcia Falconi 2008). 
Tabla 2. Puntaje para la variable empoderamiento de la mujer

\begin{tabular}{|l|c|}
\hline Dimensión & Puntaje \\
\hline Participación & 25.71 \\
\hline Temeridad & 13.16 \\
\hline Influencias externas & 16.70 \\
\hline Independencia & 10.88 \\
\hline Igualdad & 11.75 \\
\hline Satisfacción social & 13.93 \\
\hline Seguridad & 14.00 \\
\hline Puntaje Total & 106.11 \\
\hline
\end{tabular}

Fuente: Datos procesados.

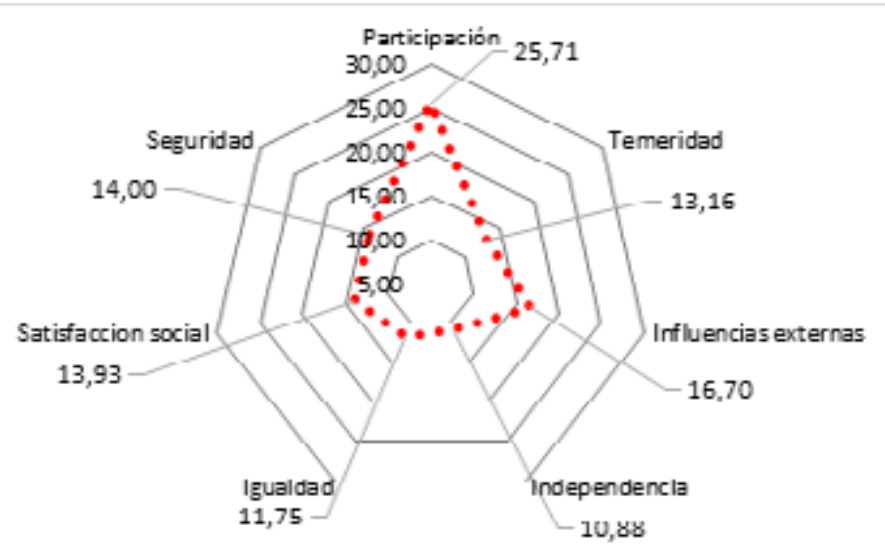

Figura 1. Nivel de empoderamiento de la mujer que pertenece a las empresas de artesanías de la región Puno

Se ha podido evidenciar el nivel alto de empoderamiento de la mujer que pertenece a las empresas de artesanía de la región Puno, resultado satisfactorio y concordante con lo afirmado por Caicedo \& Solarte (2015), quien alude al empoderamiento de la mujer, como un proceso interdependiente, complementando a lo anterior Vargas et al., (2018) y Hoinle et al., (2013) proceso donde la mujer asume la capacidad de tomar decisiones y poder ejecutarlas, autonomía económica y de decisión frente al esposo, sobre sí mismas, su cuerpo y su familia. Siendo más específicos y tomando en cuenta los resultados por dimensiones de esta variable, se ha podido evidenciar que son mujeres líderes, activas y participativas; tienden a eliminar la temeridad y dependencia de otras personas, viene desarrollando un sentido de igualdad con respecto al varón, lo que le permite competir y luchar por mejores posiciones en cualquier ámbito. Aspectos que concuerdan con la óptica de Hernández-Sanchez y García (2008). 


\section{Habilidades gerenciales que predominan en la mujer de las empresas de artesanías de la región Puno.}

Para identificar éstas, se ha medido en una escala del 1 al 5 (donde $1=$ nunca; $2=$ casi nunca; $3=$ a veces; $4=$ casi siempre y $5=$ siempre), los indicadores de las cuatro dimensiones y el promedio obtenido en el total de las encuestadas es: habilidades conceptuales (3.10), grupales (3.60), personales (3.00) y técnicas (3.70); resultado que permite inferir que las habilidades grupales y técnicas son aquellas que predominan en la mujer que pertenece a las empresas de artesanía (figura2).

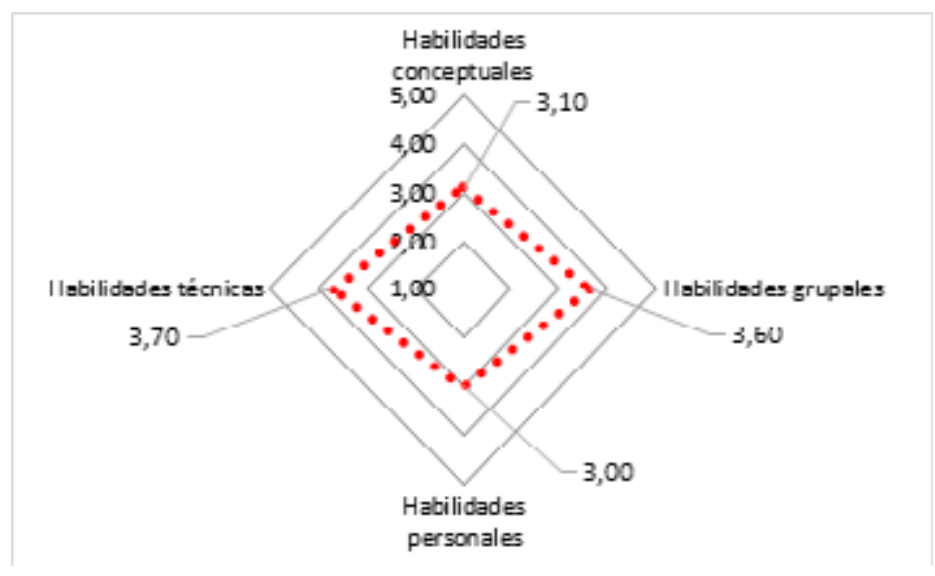

Figura 2. Habilidades gerenciales de la mujer que pertenece a las empresas de artesanías de la Región Puno

Los resultados evidencian que la mujer que pertenece a la empresa de artesanías de la región Puno ha desarrollado habilidades grupales, es decir identifican el potencial de sus colaboradoras y contribuyen a su desarrollo; dirigen, promueven y motivan equipos de trabajo para alcanzar un alto desempeño, delegando poder a sus colaboradoras y generan adecuadas relaciones públicas con grupos diversos y complejos, internos y/o externos a la organización, escenario concordante con lo afirmado por Moreno-Baquedano y Wong (2018) que afirman que no solo debe haber buenos gerentes, también líderes competentes y aptos en cualquier ámbito laboral. Por otro lado vemos que predominan las habilidades técnicas, es decir ellas tienen conocimiento del negocio y del oficio y conocen del empleo de tecnologías; al respecto Forstner (2013) afirmaba que por lo general, es la abuela o la madre tejedoras quienes regalan el arte y secretos del tejido a las nietas e hijas predilectas, así también tienen adecuada comunicación escrita y oral y establece los procesos de su arte; García-Solarte, García - Pérez de Lema y Madrid (2018) complementan, que estas habilidades, las mujeres poseen en forma natural, pues se encuentra científicamente probado que el hemisferio derecho (emociones) y el hemisferio izquierdo (conocimientos) del cerebro de una mujer se encuentran plenamente conectados. 


\section{Cumplimiento de los indicadores de responsabilidad social empresarial en las empresas de artesanías de la Región Puno}

Estos indicadores han sido medidos en una escala del 1 al $5(1=$ nunca; $2=$ casi nunca; $3=$ a veces; $4=$ casi siempre $y$ $5=$ siempre), de las siete dimensiones que propone el instrumento; el promedio obtenido en el total de las encuestadas es: valores y transparencia (1.95), público interno (0.88), medio ambiente (3.44), proveedores (1.34), clientes (2.70), comunidad (2.45) y gobierno y sociedad (1.63); resultado que permite inferir que los indicadores de las dimensiones comunidad, consumidores-clientes y medio ambiente son aquellos donde estas empresas vienen poniendo énfasis en su cumplimiento (figura 3).

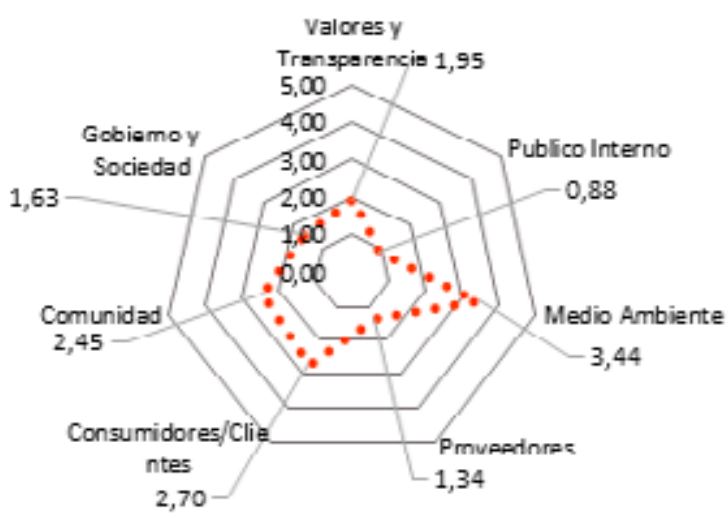

Figura 3. Cumplimiento de los indicadores de responsabilidad social empresarial de la mujer que pertenece a las empresas de artesanías de la región Puno.

En relación a la responsabilidad social empresarial se ha podido evidenciar el cumplimiento de un mayor número de indicadores en las dimensiones como comunidad, consumidores-clientes y medio ambiente, en las empresas de artesanías de la Región Puno, al respecto Rojas et al., (2017) señala que la RSE se refiere como un proceso de gestión el satisfacer las expectativas de sus diferentes grupos de interés o "stakeholders" (empleados, clientes, socios, accionistas, proveedores comunidad y medio ambiente) consideradas dimensiones Instituto Ethos (2013), Franco (2006) y IARSE (2008). Entonces se puede inferir que para las empresas de artesanías de la región Puno todavía representa una oportunidad, fortalecer el cumplimiento de indicadores de las demás dimensiones como son: valores y transparencia, publico interno, proveedores, gobierno y sociedad.

\section{Incidencia del empoderamiento de la mujer y sus habilidades gerenciales en la responsabilidad social empresarial de las empresas de artesanías de la Región Puno.}

Para determinar la incidencia de las variables empoderamiento de la mujer $\left(\mathrm{X}_{1}\right)$ y habilidades gerenciales $\left(\mathrm{X}_{2}\right)$ sobre la responsabilidad social empresarial (Y) se ha calculado el coeficiente de determinación mostrado (tabla 3 ) R2 $=0.383$ (R-cuadrado, en resultados de regresión), nos indica que la variación del empoderamiento de la mujer y sus habilidades gerenciales tienen incidencia significativa en la Responsabilidad Social Empresarial, en un 38,3 \%, se debe a las variaciones del empoderamiento de la mujer y sus habilidades gerenciales, por lo que el modelo de regresión de esta investigación es consistente. Teniendo en cuenta los resultados de la relevancia global obtenida la ecuación representativa del modelo será:

$\mathrm{RSE}=30,039+0,180 * \mathrm{HG}+0,383 * \mathrm{EM}$ 
Tabla 3. Resumen del modelo

\begin{tabular}{|c|c|c|c|c|}
\hline Modelo & $R$ & $R$ cuadrado & $\begin{array}{c}\text { R cuadrado } \\
\text { ajustado }\end{array}$ & $\begin{array}{c}\text { Error estándar } \\
\text { de la } \\
\text { estimación }\end{array}$ \\
\hline 1 &, $619 a$ &, 383 &, 375 & 8,455 \\
\hline \multicolumn{2}{l}{} \\
a. Predictores: (Constante), EM HG
\end{tabular}

Tabla 4.Análisis de varianzas ajustado al modelo ANOVA

\begin{tabular}{|l|l|l|l|l|l|l|}
\hline \multicolumn{2}{|c|}{ Modelo } & \multicolumn{1}{c|}{$\begin{array}{c}\text { Suma de } \\
\text { cuadrados }\end{array}$} & \multicolumn{1}{c|}{ gl } & \multicolumn{1}{c|}{$\begin{array}{c}\text { Media } \\
\text { cuadrática }\end{array}$} & \multicolumn{1}{c|}{ F } & Sig. \\
\hline 1 & Regresión & 6953,305 & 2 & 3476,653 & 48,635 &, $000 \mathrm{~b}$ \\
\hline & Residuo & 11223,070 & 157 & 71,485 & & \\
\hline & Total & 18176,375 & 159 & & & \\
\hline
\end{tabular}

\section{Tabla 5.Coeficientes de regresión}

\begin{tabular}{|c|c|c|c|c|c|c|}
\hline \multirow{2}{*}{\multicolumn{2}{|c|}{ Modelo }} & \multicolumn{2}{|c|}{$\begin{array}{c}\text { Coeficientes } \\
\text { no estandarizados }\end{array}$} & \multirow{2}{*}{$\begin{array}{c}\begin{array}{c}\text { Coeficientes } \\
\text { estandarizados }\end{array} \\
\text { Beta }\end{array}$} & \multirow[b]{2}{*}{$t$} & \multirow[b]{2}{*}{ Sig. } \\
\hline & & & $\begin{array}{c}\text { Error } \\
\text { estándar }\end{array}$ & & & \\
\hline \multirow[t]{3}{*}{1} & (Constante) & 30,039 & 6,543 & & 4,591 &, 000 \\
\hline & $\mathrm{HG}$ &, 180 &, 044 & ,269 & 4,123 & ,000 \\
\hline & EM &, 393 & ,053 & ,486 & 7,442 & ,000 \\
\hline
\end{tabular}

Finalmente con respecto al objetivo general de la investigación la validación del modelo recoge las vinculaciones de manera empírica y fiable de las dimensiones de la variable empoderamiento y habilidades gerenciales con la responsabilidad social empresarial de las empresas de artesanías de la región Puno, esta vinculación queda explicada mediante el coeficiente de determinación obtenido por el modelo que es $\mathrm{R} 2=0.383$, lo que significa que el $38,3 \%$ de la variabilidad de la responsabilidad social empresarial de las empresas, queda explicada por el empoderamiento de la mujer y sus habilidades gerenciales de las mujeres que las conforman. Teniendo en cuenta la Prueba de relevancia global, en esta investigación se pone de manifiesto la multidimensionalidad de la responsabilidad social empresarial a través de un nivel de empoderamiento de la mujer $\left(\mathrm{X}_{1}\right)$ y el desarrollo de las habilidades gerenciales $\left(\mathrm{X}_{2}\right)$ y su vinculación e incidencia con la responsabilidad social empresarial (Y) en las empresas de artesanías de la Región Puno.

\section{CONCLUSIÓN}

El nivel de empoderamiento de la mujer que pertenece a las empresas de artesanías de la Región Puno es alto, explicado por la suma de los promedios obtenidos en cada una de las dimensiones de la variable, con una puntuación de 106.1 1. Las habilidades gerenciales de la mujer que predominan en las empresas de artesanías de la Región Puno son habilidades conceptuales, grupales, personales y técnicas. En relación con el cumplimiento de los indicadores de responsabilidad social empresarial, las empresas de artesanía de la Región Puno vienen impulsando el cumplimiento de estos, sobre todo en aquellos que pertenecen a las dimensiones comunidad, consumidores-clientes y medio ambiente. Finalmente, con respecto al objetivo general de la investigación se concluye que en base a el coeficiente de determinación obtenido por el modelo que es $\mathrm{R} 2=0.383$, significa que el 38,3\% de la variabilidad de la responsabilidad social empresarial de las empresas, queda explicada por el empoderamiento de la mujer y sus habilidades gerenciales de las mujeres que las conforman. 


\section{REFERENCIAS}

Aparicio Valdez, Monica Rocio, y Jessica Medina Caicay . «Habilidades gerenciales que demandan las empresas en el Perú: "Un analisis comparativo”.» Lima: Universidad del Pacífico, 2015.

Ascón Villa, Jimmy Eduardo. «Teoría y Practica en el desarrollo de habilidades directivas. Un acercamiento desde la perspectiva de las instituciones de educacion superior.» Revista Ecociencia , 2018: 1-29.

BID. «Empoderamiento económico de las mujeres: Bueno para las mujeres, bueno para los negocios y bueno para el desarrollo.» II Cumbre empresarial de las Americas, 2015.

Burns Vidaurrázaga, M. «Estructuras de comercio en torno a las artesanias: La artesania en el Perú en los ultimos cuarenta años.» Artesanias de América, 2015: 18-24.

Caicedo Muñoz, Silvia Cristina, y Leonardo Solarte Pazos. «Empoderamiento de mujeres de una ONG colombiana. Un estudio de caso simple.» Revista de Administracion Pública, 2015.

CEPAL. «Lograr la igualdad de género y empoderar a todas las mujeres y las niñas en America Latina y el Caribe.» Foro de los Paises de America Latina y el Caribe sobre el Desarrollo Sostenible, 2017.

Forstner, Kathrin. «La artesanía como estrategia de desarrollo rural: el caso de los grupos de artesanas en la región de Puno (Perú).» Cuadernos de Desarrollo Rural, 2013: 141-158.

Franco Concha, Pedro. «Manual de Diagnóstico. Indicadores CGS de Responsabilidad Social Empresarial.» Atomic Energy, 2006.

Gamboa Bernal, Gilberto Alfonso. «Los objetivos de Desarrollo Sostenible: una perspectiva bioética.» Persona y Bioética (Universidad de La Sabana), 2015.

Garcia Payares, Francisco José, Efrain Arnoldo Boom Carcamo , y Schneyder José Molina Romero. «Habilidades del gerente en organizaciones del sector palmicultor en el departamento del Cesar - Colombia.»Vision de futuro, 2017: $1-21$.

Garcia Solarte, Mónica, Domingo Garcia Perez de de Lema , y Antonica Madrid Guijarro. «El género de la gerencia y la cultura organizacional de la pyme en la región de Murcia-España.» Revista Venezolana de Gerencia , 2018.

Gonzáles , Miriam R., y Judith Ley . «Habilidades gerenciales y su influencia en la competitividad de las agroempresas del valle de Mexicali, Mexico.» Revista Espacios, 2019.

Hernandez Sanchez, Julita Elemí, y Renán Garcia Falconi. Instrumento para medir el empoderamiento de la mujer. Villahermosa: Universidad Juárez Autónoma de Tabasco, 2008.

Herrera Madueño, Jesús, Manuel Larrán Jorge , y Domingo Martinez-Martinez. «Relación entre responsabilidad social y performance en las pequeñas y medianas empresas: Revisión bibliográfica.» Cuadernos de Gestión, 2013: 39-65.

Hoinle , Birgit, Rainer Ruthfuss, y Damaris Gotto . «Empoderamiento espacial de las mujeres mediante la Economía Solidaria.» Cuadernos de Desarrollo Rural, 2013: 117-139.

Instituto Argentino de Responsabilidad Social Empresarial IARSE. «Indicadores de Responsabilidad Social Empresarial.» Paso a Paso, 2008.

Instituto Ethos. «Indicadores Ethos de Responsabilidad Social Empresarial .» Journal of chemical information and modeling, 2013.

Leiva , Alma, y Joel Espejel Blanco. «Habilidades gerenciales como estrategia de competitividad empresarial en las pequeñas y medianas empresas (Pymes).» Perspectiva Empresarial, 2017: http://dx.doi.org/10.16967/rpe.

Moreno Baquedano, María Jimena, y Higinio Guillermo Wong Aitken. «Relación de las habilidad directivas y la satisfacción laboral en la empresa chicken king de Trujillo, 2018.» Cuadernos Latinoamerico de Administración (Cuadernos Latinoamericos de Administración ) XIV (2018).

Naranjo Arango, Rodrigo. «Habilidad gerenciales en los lideres de las medianas empresas de Colombia.» Pensamiento E Gestión , 2015: 119-146. 
Organización Internacional del Trabajo. «Manual de referencia sindical sobre la Agenda 2030 para el Desarrollo Sostenible.» Organización Internacional del Trabajo 1 (2017).

Rios Acuña, Sirley. «Artesanias del Peru historia tradición e innovacion.» Ministerio de Comercio Exterior y Turismo MINCETUR, 2019.

Rojas Terrazas, Maria del Rosario N., Sergio Niño Ramos, y Rosario Martinez Leon . «Responsabilidad social corporativa con perspectiva de género en el sector.» Ecorfan, 2017: 1-13.

Vargas Winstanley, Silvana, Patricia Ruiz-Bravo Lopez, y Jhonatan Clausen Lizárraga. «Empoderar para incluir: Analisis de las Multiples Dimensiones y Factores Asociados al Empoderamiento de las Mujeres en el Perú a partir del Uso de una Aproximacion de Metodologias Mixtas.» Centro de Investigacion y Desarrollo, 2018. 